\title{
LUMBAR BONE MASS PREDICTS LOW BACK PAIN IN MALES
}

HOOZEMANS M.J.M. ${ }^{1}$, KOPPES L.L.J. ${ }^{3}$, TWISK J.W.R. ${ }^{2}$, VAN DIEËN J.H. ${ }^{1}$

${ }^{1}$ Research Institute MOVE, Faculty of Human Movement Sciences, VU University Amsterdam, The Netherlands. - ${ }^{2}$ Institute for Research in Extramural Medicine (EMGO), VU University Medical Center, Amsterdam, The Netherlands. - ${ }^{3}$ TNO Quality of Life, Hoofddorp, The Netherlands

\section{Aims:}

Results of clinical and in vitro studies indicate that a fracture of the vertebral endplate, caused by compression forces, may be a primary cause of (non-specific) low back pain (LBP) (1). A logical inference from this hypothetical causative mechanism is that the compressive strength of (lumbar) vertebrae, approximated by bone mineral content (BMC) and bone mineral density (BMD), would be a determinant of LBP. The objective of the study was to investigate whether low BMC and BMD values at the age of 36 years are related to the prevalence of LBP at the age of 42 years among the study population of the Amsterdam Growth and Health Longitudinal Study.

\section{Methods:}

At the age of 36 years the lumbar BMC and BMD was determined by dual-energy x-ray absorptiometry (DEXA) in 140 males and 152 females. At the age of 42 years the participants were asked whether they had experienced LBP in the previous twelve months. Logistic regression analyses were performed to determine the relationship between BMC/BMD and LBP, adjusted for stature, bodyweight and physical activity.

\section{Results:}

$\mathrm{BMC}$ and BMD at the age of 36 years were significantly related to the reported 12-month prevalence of LBP at the age of 42 years. This relationship was only observed for males and not for females. Men within the quartile with the lowest BMC or BMD values had significant adjusted OR's for LBP of 4.21 (95\% Cl: 1.3613.0) and 3.14 (95\% Cl: 1.12-8.82) respectively, with the quartile with the highest BMC or BMD values taken as reference.

\section{Conclusion:}

In conclusion, for a male population that is not characterised by osteoporosis or old age, lower lumbar BMC and BMD values are associated with a higher risk of reporting LBP in the future. Combined with the relationship between physical activity and bone mass (2), these findings support physical activity, in particular weight-bearing activity, as a preventive measure to decrease the risk of developing LBP.

Keywords: Biomechanics, Epidemiology, Back, Low back

\section{References:}

Van Dieën $\mathrm{JH}$, Weinans $\mathrm{H}$, Toussaint HM. Fractures of the lumbar vertebral endplate in the etiology of low back pain. A hypothesis on the causative role of spinal compression in a-specific low back pain. Medical Hypotheses. 1999;53(3):246-52.

Bakker I, Twisk JW, Van Mechelen W, Roos JC, Kemper HC. Ten-year longitudinal relationship between physical activity and lumbar bone mass in (young) adults. J Bone Miner Res. 2003;18(2):325-32. 